\title{
Denoising of Rain Streak Hd Videos Using Hevc in Convolution Neural Network
}

\author{
R.Mohanapriya ${ }^{1 *}$, Dr. C.Gowri Shankar ${ }^{2}$ \\ ${ }^{*}$ Student Member, Department of EEE, KSR College Of Engineering, Namakkal, India \\ ${ }^{2}$ Pro fessor, Department of EEE, KSR College of Engineering, Namakkal, India
}

*Corresponding Author: R. Mohanapriya, Student Member, Department of EEE, KSR College of Engineering, Namakkal, India

\begin{abstract}
In the rainy season most of the images are cannot able to see clearly, sometimes it may also lead to do crime. So inorder to avoid that problem this project is done to see the images clearly in the rain also. The aim of this project is to remove the rain streaks from the HD videos using the algorithm of convolution neural network $(C N N)$. It is done using MATLAB. First the videos are converted into frames and the frames are converted into the images. Convolution neural network is used to remove the unwanted noises. Thus the same the unwanted rain streaks are removed. The input is given as the rain streak images, using the convolution neural network algorithm the rain streaks are removed and the output comes without the rain streak images. Such a new technique makes the proposed method capable of properly extracting rain streaks from videos, thus getting fine video with deraining effects. And the particular part can also be shown clearly while the car is in the rain. For example car number plate can be taken, if the car is travelling in the rain, the number plate cannot be detected in the normal camera, using the CNN algorithm the number plate of the car can be detected in the clear manner.
\end{abstract}

Keywords: Machine Learning, convolution neural network

\section{INTRODUCTION}

Chuang and Chan [3] had implemented the proposed system of "A Novel Video Summarization Method for Multi- Intensity Illuminated Infrared Videos," IEEE Int'l Con! On Multimedia and Expo (ICME), San Jose, USA. In nighttime video police work, correct illumination plays a key role for the image quality. For normal IR-illuminators with mounted intensity, faraway objects are usually laborious to spot thanks to shy illumination whereas near objects could suffer from over-exposure, leading to image foreground/background of poor quality. During this paper we have a tendency to planned a completely unique video summarization technique that utilizes a novel multi-intensity IRilluminator to get pictures of human activities with completely different illumination levels. By adopting GMM-based foreground extraction procedure for pictures nonheritable for every illumination level, foreground objects with most plausible quality will be elect and united with a preselected illustration for still background. The result brings out an affordable video outline for moving foreground that is usually unattainable for nighttime police work videos.

Chuang et al. [3] have enforced the projected system of "MI3: Multi-Intensity Infrared Illumination Video info," IEEE Int'l Conf on Visual Communications and Image process (VCIP), Singapore, Singapore, Vision-based video police investigation systems have gained increasing quality. However, their practicality is well restricted beneath nighttime conditions because of the poor visibility caused by improper illumination. Equipped on scotopic vision cameras, standard infrared (IR) illuminators of fixed-intensity sometimes cause the imaging downside of overexposure (or underexposure) once the thing is simply too near (or too so much from) the camera. to beat this limitation, we tend to use a completely unique multi-intensity IR illuminator to increase the effective vary of distance of camera police investigation, Associate in Nursing establish during this paper the MI3 (Multi-Intensity Infrared Illumination) info supported such an illuminator. The info contains intensity variable video sequences of many indoors and out of doors scenes. Ground truths together with individuals enumeration and foreground labeling are provided for various analysis usages. Performances of connected algorithms are tested for demonstration and analysis. 
Sullivan et al. [6] have enforced the projected system of "Overview of the high potency video committal to writing (HEVC) normal. High potency Video committal to writing (HEVC)" is presently being ready because the newest video coding normal of the ITU-T Video committal to writing specialists clusters and therefore the ISO/IEC film specialists cluster. the most goal of the HEVC standardization effort is to modify considerably improved compression performance relative to existing standards-in the vary of fifty bit-rate reduction for equal sensory activity video quality. This paper provides an summary of the technical options and characteristics of the HEVC normal.

Miiller and Wang [7] had enforced the projected system of "Overview of the Multi-view and 3D Extensions of High potency Video committal to writing," IEEE Trans. on Circuits and Systems for Video Technology (CSVT), vol. 26, Issue 1, pp. 35-49. The High potency Video committal to writing (HEVC) normal has recently been extended to support economical illustration of multi read video and depth-based 3D video formats. The multi read extension, MV-HEVC, permits economical committal to writing of multiple camera views and associated auxiliary photos, and might be enforced by reusing single-layer decoders while not ever-changing [10] the block-level process modules since block-level syntax and decipherment processes stay unchanged. Bit rate savings compared with HEVC broadcast are achieved by enabling the utilization of inter-view references in motion-compensated prediction. The a lot of advanced 3D video extension, [11]3D-HEVC, targets a coded illustration consisting of multiple views and associated depth maps, PRN for generating extra intermediate views in advanced 3D displays. Extra bit rate reduction compared with MV-HEVC is achieved by specifying new blocklevel video committal to writing tools that expressly exploit applied mathematics dependencies between video texture and depth and specifically adapt to the properties of depth maps.

Chen et al. [3] have implemented the proposed system of New Application of MV - and 3D-HEVC for Multi-Intensity Illuminated Infrared Video Coding," IEEE International Conference on Visual Communications and Image Processing (VCIP), Chengdu, China, are the extensions of High Efficiency Video Coding (HEVC) originally designed for Multi-view and 3D video coding respectively. In this paper we reveal a new application of using these video coding standards, i.e., to compress the Multi-Intensity Illuminated Infrared (MIIR) video, a new type of infrared video developed to overcome the limitation of improper illumination conditions for nighttime surveillance. With the proposed encoding method for the MIIR video, the coding structures of MV- and 3D-HEVC can compress such video in a more efficient way, i.e., up to $13.2 \% / 17.6 \%$ bitrate reductions with MV/3D-HEVC compared with standalone [9] HEVC compression and 64.4\%/68.7\% bitrate reductions compared with simulcast HEVC coding. Experiment results also reveal that weighted prediction algorithm in HEVC can be improved to deal with large change of brightness in the MIIR video.

\subsection{Matlab Working}

MATLAB can interface with other languages can call functions and subroutines written in the programming languages $\mathrm{C}$ or FORTRAN. A wrapper function is created allowing MATLAB data types to be passed and returned. MEX files (MATLAB executables) are the dynamically loadable object files created by compiling such functions. Since 2014 increasing two-way interfacing with Python was being added.

MATLAB supports developing applications with graphical user interface (GUI) features.

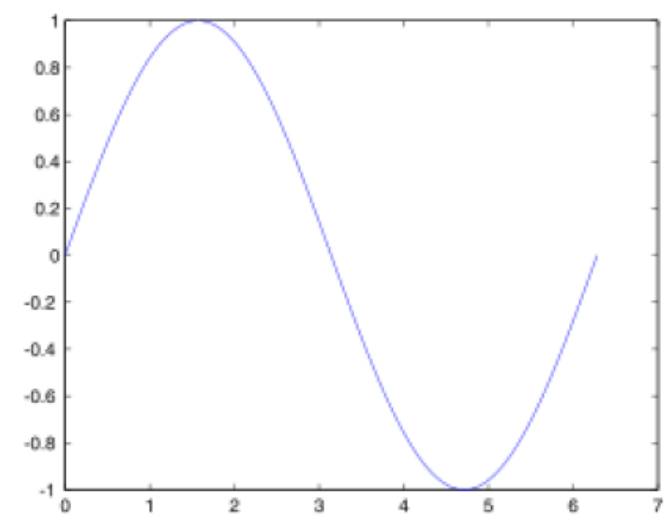

Figure1 (A). Dimensional Image of GUI 
Figure 1 A MATLAB program can produce three-dimensional graphics using the functions surf, plot3 or mesh. For example

$$
\begin{gathered}
\mathrm{x}=0 \text { :pi } / 100: 2 * \mathrm{pi} ; \\
\mathrm{y}=\sin (\mathrm{x}) ; \\
\mathrm{Plot}(\mathrm{x}, \mathrm{y})
\end{gathered}
$$

\section{RELATED WORKS}

A wrapper perform is formed permitting MATLAB knowledge sorts to be passed and came back. MEX files (MATLAB executables) are the dynamically loadable object files created by aggregation such functions. Since 2014 increasing two-way interfacing with Python was being extra.

MATLAB supports developing applications with graphical interface (GUI) options. Miiller and Wang [7] had enforced the projected system of "Overview of the Multi-view and 3D Extensions of High potency Video cryptography," IEEE Trans. on Circuits and Systems for Video Technology (CSVT), vol. 26, Issue 1, pp. 35-49. The High potency Video cryptography (HEVC) normal has recently been extended to support economical illustration of multi read video and depth-based 3D video formats. The multi read extension, MV-HEVC, permits economical cryptography of multiple camera views and associated auxiliary photos, and might be enforced by reusing single-layer decoders while not dynamical the block-level process modules since block-level syntax and coding processes stay unchanged. Bit rate savings compared with HEVC broadcast are achieved by enabling the employment of inter-view references in motion-compensated prediction. The a lot of advanced 3D video extension, [8]3D-HEVC, targets a coded illustration consisting of multiple views and associated depth maps, PRN for generating extra intermediate views in advanced 3D displays. extra bit rate reduction compared with MV-HEVC is achieved by specifying new block-level video cryptography tools, that expressly exploit applied mathematics dependencies between video texture and depth and specifically adapt to the properties of depth maps.

Chen et al. [3] have enforced the projected system of recent Application of MV - and 3D-HEVC for Multi-Intensity well-lighted Infrared Video cryptography," on Visual Communications and Image process (VCIP), Chengdu, China, are the extensions of High potency Video cryptography (HEVC) originally designed for Multi-view and 3D video coding severally. during this paper we have a tendency to reveal a replacement application of mistreatment these video cryptography standards, i.e., to compress the Multi-Intensity well-lighted Infrared (MIIR) video, a replacement variety of infrared video developed to beat the limitation of improper illumination conditions for nighttime police investigation. With the projected cryptography technique for the MIIR video, the cryptography structures of MV- and 3D-HEVC will compress such video during a a lot of economical approach, i.e., up to $13.2 \% / 17.6 \%$ bitrate reductions with MV/3D-HEVC compared with standalone HEVC compression and sixty four.4\%/68.7\% bitrate reductions compared with broadcast HEVC cryptography. Experiment results conjointly reveal that weighted prediction algorithmic rule in HEVC will be improved to accommodate massive amendment of brightness within the MIIR video.

\section{HEVC IN CNN}

Convolution neural network (CNN or Conv.Net) could be a category of deep, feed-forward artificial neural networks, most typically applied to analyzing visual imagination. CNNs use a variation of multilayer perceptrons designed to want tokenish preprocessing. They're conjointly called shift invariant or area invariant artificial neural networks (SIANN), supported their shared-weights design and translation unchangeableness characteristics.

A CNN consists of associate input and an output layer, moreover as multiple hidden layers. The hidden layers of a CNN usually accommodate convolutional layers, pooling layers, absolutely connected layers and normalization layers. Description of the method as a convolution in neural networks is by convention. Mathematically it's a cross-correlation instead of a convolution. This solely has significance for the indices within the matrix, and therefore that weights are placed at which index. Convolutional layers apply a convolution operation to the input, passing the result to the subsequent layer. The convolution emulates the response of a private vegetative cell to visual stimuli. 
High potency Video cryptography, conjointly called H.265 and MPEG-H half two, could be a video compression normal, one in every of many potential successors to the wide used AVC. Compared to AVC, HEVC offers concerning double the info compression quantitative relation at the identical level of video quality, or considerably improved video quality at the identical bit rate. It supports resolutions up to $8192 \times 4320$, together with $8 \mathrm{~K}$ UHD.

The main goals of HEVC style embody enlarged video resolutions and therefore the exploitation of multiprocessing architectures. HEVC is fitted to a spread of applications, like broadcast of high definition (HD) TV signals over satellite, terrestrial transmission systems and cables, video content acquisition and redaction systems, security applications, camcorders, Blue-ray discs, net and mobile network video and time period colloquial applications that embody video conferencing, video chat, and tele-presence systems .

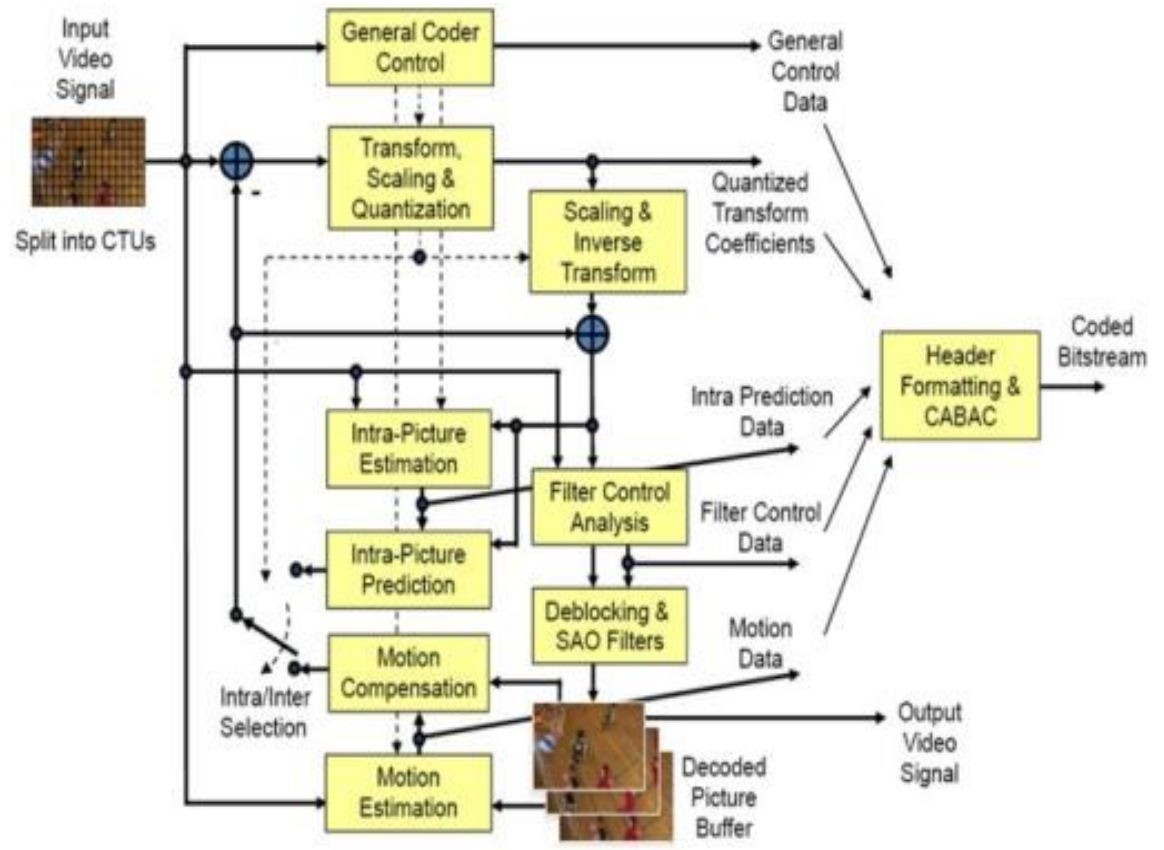

Figure1 (B). Functions of HEVC

Figure1 (B): Functions of HEVC Explains the Functions of HEVC that are Explained Below in Step by Step Method

1. HEVC introduces the 3 new ideas of cryptography Unit (CU), Prediction Unit (PU) and remodel Unit (TU).

2. The cryptography pipeline splits every frame into what are referred to as cryptography Tree Units (CTU). A CTU has one cryptography Tree Block (CTB) covering a luma block and therefore the corresponding intensity blocks. The scale of luma, L, could see sixty four, thirty two or sixteen samples.

3. A CTB may be divided into smaller blocks employing a Quad Tree structure. A given copper may be a part of CTB and might be divided recursively into four CUs and every has an associated division into Prediction Unit (PU) and remodel Unit (TU).

4. Atomic number 94 is made once a prediction methodology is chosen. The knowledge of the prediction methodology (Intra/Inter and therefore the connected data) is contained within the atomic number 94.

5. The Prediction Block may be split employing a sampling theme that will vary $64 \times 64$ to $4 \times 4$ samples.

6. For the intra-prediction in HEVC, thirty three angular directions are used.

7. The atomic number 94 may be sub divided into two rectangular or 4 sq. partitions, within the interprediction. For motion compensation, the atomic number 94 division could also be unidirectional or bi-directional. 
8. HEVC uses remodel cryptography of the prediction residual within the similar means as its forerunner H.264/AVC. The residual block is split into smaller sq. remodel blocks (TBs).

9. The remodel is associate approximation of DCT its block sizes may be $32 \times 32,16 \times 16,8 \times 8$ and $4 \times 4$.

10. HEVC conjointly has mode dependent different remodel. Another whole number remodel derived from separate sin transform (DST) is applied on every TB of size $4 \times 4$. The DST is merely applied on luma remodel blocks.

11. Remodel coefficients within the encoder facet are measure to limit the amount of bits. At the beginning, the quantization level is outlined by a quantization parameter (QP) worth that controls the uniform-reconstruction quantization (URQ) theme. To any decrease the bitrate, the QTCs are entropy coded.

12. Just one entropy cryptography is laid out in the HEVC, i.e. the context adaptive binary arithmetic cryptography (CABAC). The CABAC is employed to write in code the primary coefficients (levels), Golomb-Rice cryptography to code the subsequent and Exponential-Golomb coding is utilized for cryptography the last levels.

13. The degradation of frames, that is caused by compression, is repaired by applying 3 varieties of filters specifically the sample adaptive offset (SAO), de-blocking and adaptive loop filters (ALF).

14. A brand new video parameter set (VPS) is additionally introduced in HEVC.

15. To extend the multiprocessing capability, HEVC introduces 3 new options aside from the slices, like tiles, wave front multiprocessing (WPP) and dependent slices.

A. Pixel:

Image process may be a set of the electronic domain whereby the image is born-again to associate array of little integers, referred to as pixels, representing a physical amount like scene radiance, keep in a very digital memory and processed by pc or different digital hardware.

Suppose we have a tendency to take a picture, a photo, say. For the instant, lets create things straightforward and suppose the picture is black and white (that is, plenty of reminder grey), thus no color. We have a tendency to could think about this image as being a 2 dimensional operate, wherever the operate values offer the brightness of the image at any given purpose, as shown in figure one.2. we have a tendency to could assume that in such a picture brightness values may be any real numbers within the vary zero.0 (black) to one.0 (white). The ranges of $\mathrm{x}$ and $\mathrm{y}$ can clearly rely on the image, however they will take all real values between their minima and maxima.

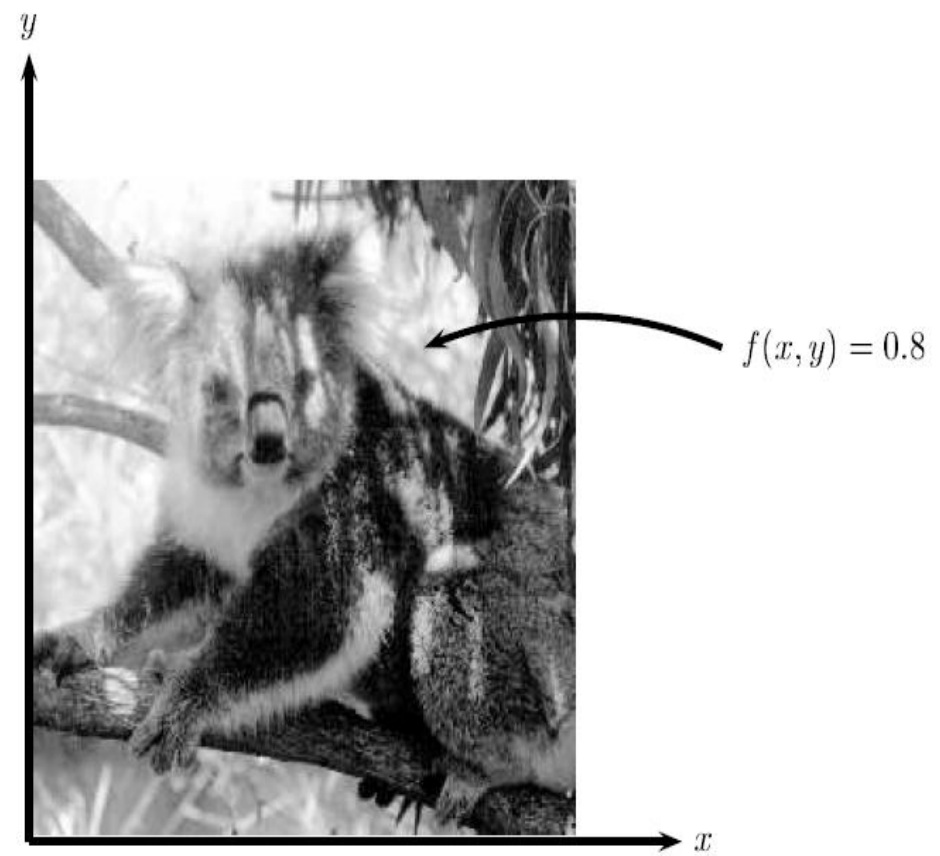

Figure2. A Grey Scale Image 


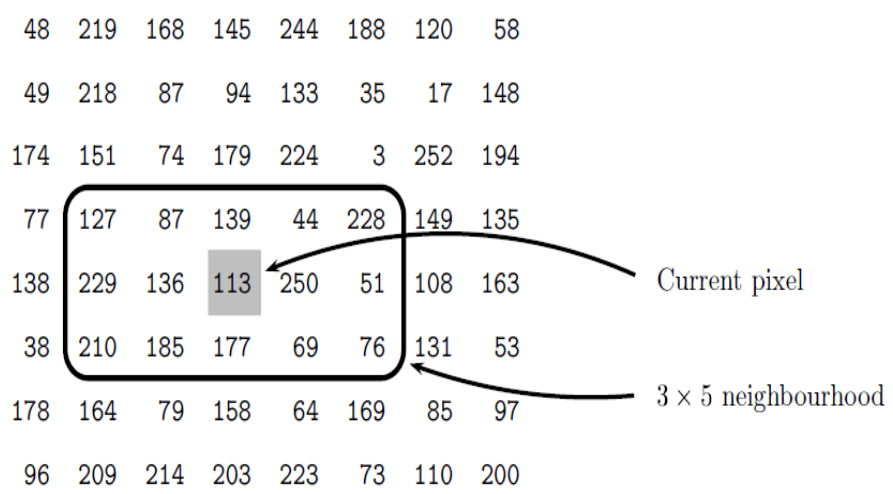

Figure3. Pixel Representation

Unsupervised learning, on the other hand, the training examples give by the system is not labelled with the belonging class [5]. So the system develops and organizes the data, searching usual characteristics among them, and changing based on inner knowledge.

\section{B. Working Principle}

i) Open the MATLAB software and extract the file.

ii) Rain affected Video is given as the input to the system, videos are converted into frames, frames are converted into images.

iii) Images and the rain streaks are get separated using the sampling process.

iv) Using Convolution neural network algorithm $(\mathrm{CNN})$, the rain streaks are removed and the high quality HD video are obtained.

v) The PSNR value can be detected in the output. (Fig 3.3).

\section{Encoding Methods}

After separating the raw MIIR video into sub-videos (channels) as described in the previous section, the split videos can be arranged as if they are obtained from different sources (views) to fit the desirable video coding framework for efficient compression. In this paper, we adopt the latest MVHEVC and 3D-HEVC standards, which are extensions of the single-view HEVC for multiple views/cameras, to compress the "multi-channel" version of the MIIR videos. Details of the related coding schemes will be described in following subsections.

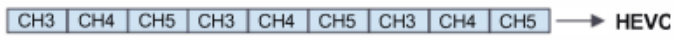

(a) Plain HEVC encoding of raw MIIR video

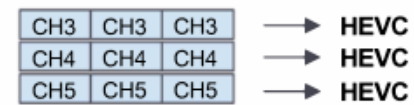

(b) Simulcast HEVC encoding for each channel

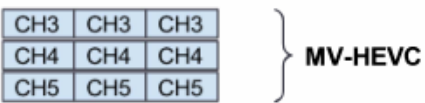

(c) Multi-view encoding arrangement for multiple channels

\begin{tabular}{l|l|l|}
\hline $\mathrm{CH} 3$ & $\mathrm{CH} 3$ & $\mathrm{CH} 3$ \\
\hline $\mathrm{CH} 4$ & $\mathrm{CH} 4$ & $\mathrm{CH} 4$ \\
\hline $\mathrm{CH} 5$ & $\mathrm{CH} 5$ & $\mathrm{CH} 5$ \\
(d) 3D mode encoding arrangement with dummy depth map for \\
multiple channels
\end{tabular}

Figure4. MV-HEVC and 3D-HEVC

\section{Mv-HeVC AND 3D-HeVC ENCODING}

In addition to applying broadcast secret writing to use the brightness similarity of frames at intervals the identical channel, image frames in close channels can also be used for prediction since their time instances are a lot of close ( \pm 1 inclose image capturing) to the present frame compared with the 
previous/next frame in the identical channel ( \pm six frames for a 6-channel MIIR video), although close channels can have completely different brightness levels. For MV-HEVC and 3D-HEVC, as introduced in Sec. I, video frames of various views are encoded at the identical time with the interview prediction enabled. Therefore, to adopt MV-HEVC and 3D-HEVC for additional improvement of the compression performance of MIIR videos, we have a tendency to projected to rearrange every channel of MIIR video as a special read within the corresponding committal to writing frameworks. So the inter-view predictions are custom-made as "inter-channel" prediction for the MIIR video to enhance the compression performance.

Compression performance of different coding setting, (Channel 3,4,5 are test and the anchor is plain HEVC coding)

Table1. Comparision Performance

\begin{tabular}{|l|l|l|l|l|l|}
\hline Test video clip & MV HEVC & 3D-HEVC & 3D-HEVC IC & 3D-HEVC IC+WP & 3D-HEVC IC+QP \\
\hline No Person & $-13.2 \%$ & $-13.5 \%$ & $-18.9 \%$ & $-12.2 \%$ & $-18.5 \%$ \\
\hline One Person & $-12.7 \%$ & $-12.9 \%$ & $-16.7 \%$ & $-12.5 \%$ & $-16.2 \%$ \\
\hline Multiple Persons & $-9.5 \%$ & $-9.9 \%$ & $-13.0 \%$ & $-9.7 \%$ & $-13.0 \%$ \\
\hline Bus & $-11.9 \%$ & $-12.2 \%$ & $-16.6 \%$ & $-14.9 \%$ & $-17.5 \%$ \\
\hline Stair & $-0.5 \%$ & $-0.4 \%$ & $-3.4 \%$ & $-4.8 \%$ & $-6.8 \%$ \\
\hline Average & $-9.56 \%$ & $-9.78 \%$ & $-13.72 \%$ & $-10.82 \%$ & $-14.40 \%$ \\
\hline
\end{tabular}

\section{RESULTS AND DISCUSSION}

The simulation is done in the MATLAB and the output videos are processed without the rain streaks. PSNR value is obtained and the rain removal percentage is shown in the command window.

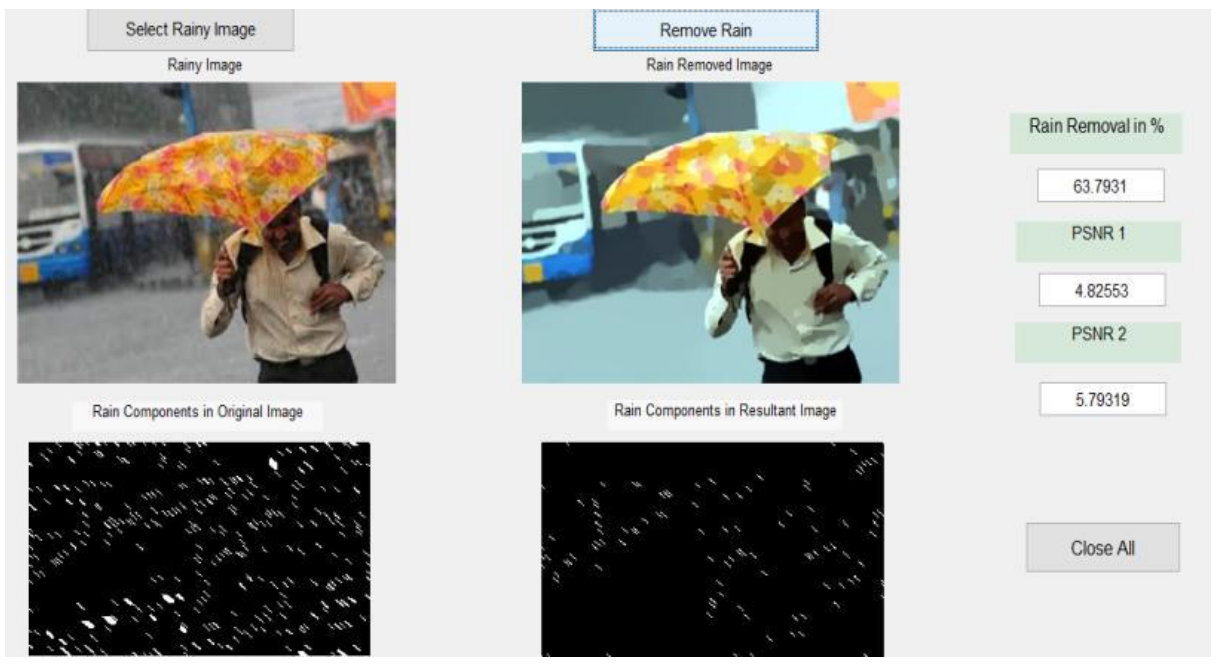

Figure5: Simulation Output 1

The rainy image is selected, rain streaks get separated and the image without rain is obtained. The value of PSNR to the input image is 4.825 and the value of PSNR to the output image is 5.793. (Figure5).

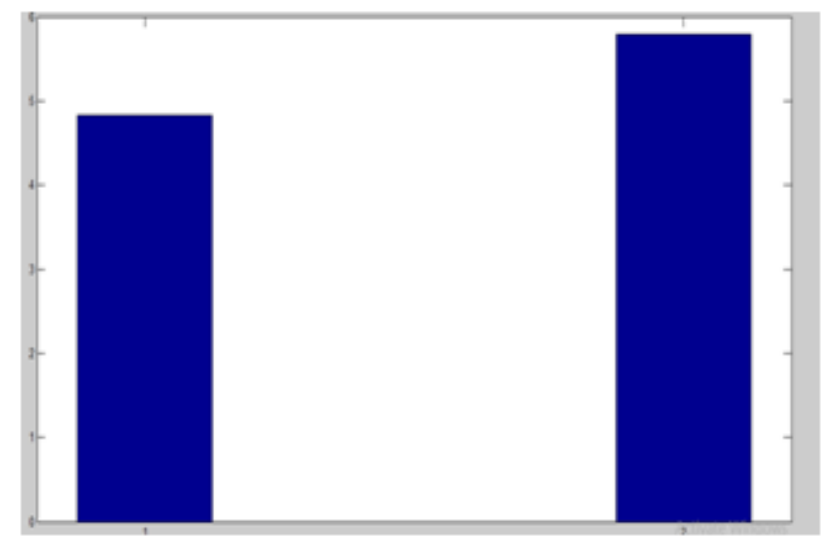

Figure6. Graphical Representation for Output 1 
The graphical representation shows the difference between the input and output images. (Figure6).

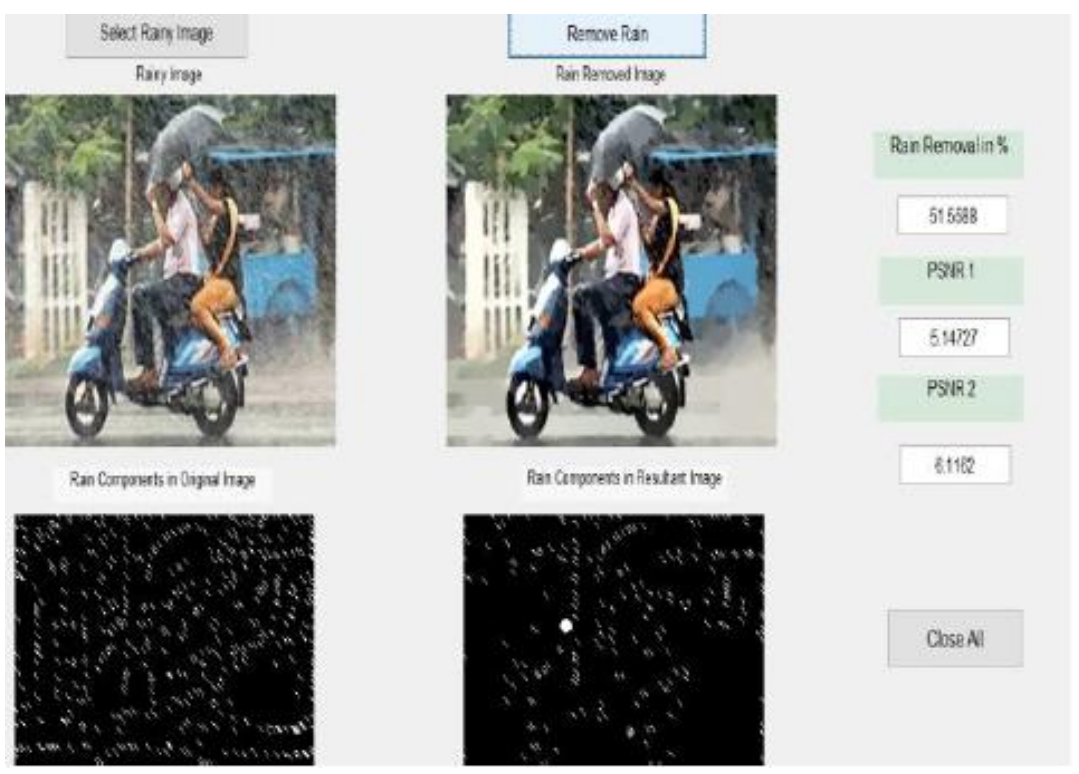

Figure7: Simulation Output 2

The rainy image is selected, rain streaks get separated and the image without rain is obtained. The value of PSNR to the input image is 5.147 and the value of PSNR to the output image is 6.116. (Figure7)

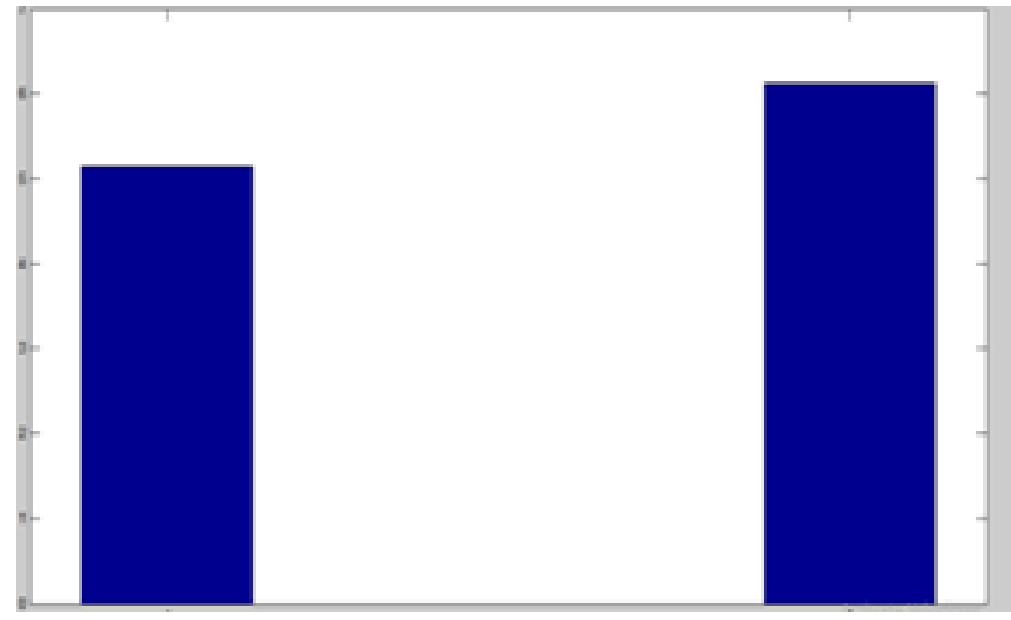

Figure8. Graphical Output for Output 2

The graphical representation shows the difference between the input and output images. (Figure8).

\section{CONCLUSION AND FUTURE WORK}

The main objective of this project is to get rid of the unwanted noise (rain affected video) within the HD videos. IT'S clearly mentioned with the proof that the MATLAB is that the safe tool for removing the rain streaks. From the MATLAB the PSNR price is additionally obtained and also the rain removal share for the input image and the output image is also obtained within the command window. In my future work, the applying work is completed. Associate in Nursing example \} if an automobile image is taken, if the automobile travels throughout rainy acquisition means that the amount plate of the car won't be clearly seem within the traditional camera. Thus inorder to indicate the amount plate of the automobile in a very clear manner exploitation CNN the longer term work is completed.

\section{REFERENCES}

[1] Teng (2010) "A New Design of IR Illuminator for Nighttime Surveillance," MS Thesis, National Chiao Tung Univ.

[2] Chuang and Lin,(2011) "Intelligent Nighttime Video Surveillance Using Multi-Intensity Infrared Illuminator," Proc. of the World Congress on Engineering and Computer Science, vol. 11. 
[3] Chen and Chuang (2012) "Robust License Plate Detection in Nighttime Scenes Using Multiple Intensity IR-Illuminator,” IEEE Int'1 Symposium on Industrial Electronics, pp. 93-898.

[4] Chuang and Tsai (2013) "A Novel Video Summarization Method for Multi- Intensity Illuminated Infrared Videos," IEEE Int'l Con! On Multimedia and Expo (ICME), San Jose, USA.

[5] Teng and Liu (2015) "MI3: Multi-Intensity Infrared Illumination Video Database," IEEE Int'l Conf on Visual Communications and Image Processing (VCIP), Singapore, Singapore.

[6] Sullivan and Wiegand (2012) "Overview of the High Efficiency Video Coding (HEVC) Standard," IEEE Trans. on Circuits and Systems for Video Technology (CSVT), vol. 22, no. 12, pp.I649-1668.

[7] Miiller and Wang (2015) "Overview of the Multiview and 3D Extensions of High Efficiency Video Coding," IEEE Trans. on Circuits and Systems for Video Technology (CSVT), vol. 26, Issue 1, pp. 35-49.

[8] Teng, and Chuang (2016) "New Application of MV - and 3D-HEVC for Multi-Intensity Illuminated Infrared Video Coding," IEEE In!'l Conf on Visual Communications and Image Processing (VCIP), Chengdu, China.

[9] Teng and Chuang (2015)"A Novel Synchronous Multi- Intensity IR Illuminator Hardware Implementation for Nighttime Surveillance," SPIE Proc. of Video Surveillance and Transportation Imaging Applications 20J 5, San Francisco, USA,

[10] "The HEVC software repository," [Online] https:llhevc.hhi.fraunhofer.de/svnlsvn_HEVCSoftware.

[11] F. Bossen, "Common HM Test Conditions and Software Reference Configurations," document of Joint Collaborative Team on Video Coding, JCTVC-LIIOO, Genev Switzerland, Jan. 2013.

Citation: R.Mohanapriya, (2019). "Denoising of Rain Streak Hd Videos Using Hevc in Convolution Neural Network". International Journal of Innovative Research in Electronics and Communications (IJIREC), 6(1), pp.7-15. DOI: http://dx.doi.org/10.20431/2 349-4050.0601002

Copyright: (C) 2018 Authors. This is an open-access article distributed under the terms of the Creative Commons Attribution License, which permits unrestricted use, distribution, and reproduction in any medium, provided the original author and source are credited. 